\title{
Histoire d'une représentation restrictive. Portrait du cadre en professionnel contrarié
}

\author{
Eve Lamendour \\ Université de Nantes, LEMNA
}

\begin{abstract}
Résumé
La diffusion de la fonction administrative dans l'entreprise, malgré le zèle de Fayol dès le début du XX $\mathrm{XX}^{\mathrm{e}}$ siècle, semble avoir buté contre l'image d'un rôle apparemment superflu. Nous proposons d'examiner quelle a été cette image en partant de la représentation de la fonction managériale à travers un art populaire, le cinéma. A partir de 1914, on observe une évolution significative de l'image du monde du travail : apparaissent à l'écran la complexité nouvelle de l'entreprise et sa professionnalisation.

L'image des cadres administratifs pour cette période tend à surenchérir le caractère malaisément définissable de leur rôle. Statiques, ces personnages ne semblent pas à même d'intervenir dans l'entreprise. L'échec possible, anticipé, paraît un trop grand risque pour leur permettre de s'engager dans l'action. Nous nous appuyons sur l'analyse de films mettant en scène des cadres pour mieux saisir la nature de cette sclérose par peur de l'échec.
\end{abstract}

\section{Mots clefs}

Management, représentation, histoire des organisations, échec, risque.

\section{Remerciements}

Cette recherche a été possible grâce à l'accès privilégié à leurs fonds que nous ont accordé le Forum des Images, les Archives Françaises du Film - CNC et les Archives Gaumont-Pathé. Qu'ils en soient ici chaleureusement remerciés. Nous devons aussi remercier Henri Bousquet, éditeur des catalogues Pathé, pour ses précieuses indications et sa relecture commentée de notre corpus.

Notice biographique

Eve Lamendour est docteur en sciences de gestion, chercheuse associée au Laboratoire d'Economie et de Management de Nantes Atlantique. Sa recherche porte sur les représentations du management et sur l'histoire des organisations. Usant d'une approche pluridisciplinaire (gestion, histoire, analyse filmique) dans sa thèse, elle a étudié la représentation du management dans le cinéma français de 1895 à 2005 . Elle s'intéresse actuellement au management tel qu'il apparaît à travers l'édition française et anglo-saxonne.

\section{eve.lamendour@univ-nantes.fr}




\title{
History of a partial representation. Portrait of the executive as a restricted professional
}

\begin{abstract}
My objective is to try to understand why, despite the enthusiasm shown by Henri Fayol at the beginning of the $20^{\text {th }}$ century; the spread of the administrative function in the enterprise has come up against the image of a profession that is superfluous. I propose to analysis what precisely this image was while focusing on popular culture, i.e. features films. A significant transformation in this representation is to be noticed starting from 1914: the work place became more complex, filled with newcomers.

The image of the executives was still indistinct: on screen they are recognizable but they don't act. Their reluctance to engage in any managerial situation is due to their fear of making a mistake, or worse, their fear of failure. We want to use the cinematographic material to better understand why the fear of failure wasn't recognized as failure itself and how it has transformed into a state of complete ossification of the profession.
\end{abstract}

\section{Keywords}

Management, representation, organizational history, failure, risk aversion 


\section{Histoire d'une représentation restrictive. Portrait du cadre en professionnel contrarié}

\section{Introduction : Des mots et des images pour une fonction balbutiante}

La diffusion de la fonction administrative dans l'entreprise, malgré le zèle de Fayol dès le début du siècle, semble avoir buté contre l'image d'un rôle apparemment superflu. La notion de cadre administratif commence seulement à faire son apparition dans les années 1930. A propos du terme de cadre, Luc Bolstanski, dans son étude sur cette profession comme groupe social constitué, indique que «le mot commence à être utilisé durant la période [les années 1930] mais ne s'imposera que sous Vichy»(Bolstanski, 1982, p.66). Le terme de cadre, devenu transparent pour un français en ce début de $\mathrm{XXI}^{\mathrm{e}}$ siècle, est malaisé à traduire avec justesse dans une autre langue. Si nous examinons deux cultures proches géographiquement, celles de l'Italie et de la Grande-Bretagne, nous découvrons un univers en apparence proche dans une autre langue latine et une perspective radicalement différente dans la langue anglaise. Le quadro italien paraît l'exact équivalent de cadre, mais renvoie par son étymologie à l'image dont on ne peut détacher le regard ; il s'agit moins du cadre qui délimite l'œuvre que du tableau lui-même. L'origine de quadro réfère aussi au cahier qui permet de tenir une liste (Sakhno, 2001) et semble évoquer alors une fonction orientée vers l'archive et l'enregistrement. L'executive anglais est directement engagé dans l'action, to execute exprime l'action de réaliser, d'accomplir. On comprend les réticences du traducteur quand l'homologue français de l'executive et du quadro s'applique à fixer les limites et à encadrer ses subordonnés, si l'on s'en tient à la seule étymologie.

Nous proposons d'approfondir ce qui pourrait être une spécificité française et d'examiner quelle a été l'image de ce groupe professionnel. Il s'agit donc d'interroger l'image des porteurs de la fonction administrative et de ne pas nous restreindre aux seuls bénéficiaires du titre de cadre. Notre recherche porte sur la représentation de la fonction managériale à travers le 
cinéma français depuis 1895, mais la période allant de 1914 à 1947 nous retiendra particulièrement car elle témoigne d'une représentation du management assez unifiée en dépit des soubresauts et conflits qui marquent l'époque. Sans nier la variété et l'évolution des pratiques managériales, nous voudrions ici nous attacher aux points de convergence que présentent les évocations filmiques.

Durant cette période, l'entreprise dans la fiction change et prend de l'ampleur. Ce qui se joue dans ce moment de la production cinématographique est, en premier lieu, un goût relativement nouveau pour le monde de l'entreprise. Valorisé à travers des usines monumentales, une architecture avant-gardiste, un personnel pléthorique, cet univers donne lieu à des films soucieux d'actualité et comme ajustés - pour la majorité d'entre eux - sur le moment contemporain, qu'ils aient été réalisés à partir de scénarios originaux ou basés sur l'adaptation actualisée de romans ou pièces de théâtre. Ainsi, en 1928, Marcel L'Herbier réalise avec L'Argent une version ancrée dans le présent du roman écrit par Zola entre 1890 et 1891. Dans cet ensemble de films, la représentation qui est donnée des usines, banques ou manufactures, fait apparaître un changement de conception de ce que peut être une entreprise. Ce changement marque une rupture par rapport aux deux premières décennies du cinéma. Une complexité inédite du monde du travail s'impose à l'écran ; elle est notamment visible dans la montée de certaines fonctions : comptables, employés, cadres administratifs, actionnaires et conseils d'administration. Et la manière enrichie de penser l'entreprise se traduit à l'écran par une démonstration de la professionnalisation progressive du management. La question est alors, pour nous, de comprendre comment, dans un tel contexte, le cadre administratif se voit doté par la fiction d'un caractère statique qui le laisse incapable d'intervenir dans l'entreprise. Pour remplir ce programme, il nous faut en premier lieu définir la place dévolue à la fonction administrative dans la représentation filmique. Nous verrons ensuite comment cette fonction est mise en concurrence et chercherons à identifier le porteur légitime du rôle managérial. 
Puis, nous reviendrons sur les attributs du cadre afin de comprendre l'échec que la scène

filmique lui associe avec une certaine systématique.

\section{Encadré n 1 - Méthodologie}

Un renouvellement dans l'approche d'une question classique peut parfois être obtenu par un décalage du regard. En abordant le management par l'histoire, nous pensions sortir de «la mentalité de l'initié » évoquée par Marc Bloch (1967, p.32) pour apporter une perspective que nous voudrions renouvelée sur les hommes porteurs de la fonction managériale. Sur cette question de gestion, notre approche est donc historique quand notre corpus relève du champ cinématographique.

\section{Corpus}

Notre recensement a été établi à partir de catalogues de films français. Les informations issues des catalogues génériques concernant les films produits en France ont été recoupées avec les données des catalogues reconstitués des firmes de production Pathé, Gaumont et Eclair. Pour la période 1914-1947, nous avons identifié un corpus de 126 films de fiction français mettant en scène des personnages exerçant la conduite d'une entreprise privée. Nous avons retenu les films donnant à ce thème une place de choix dans le récit ou lui accordant une représentation particulièrement intéressante bien que mineure.

\section{Mode d'analyse}

L'analyse qui suit a porté sur les films que nous avons pu visionner dans cet ensemble (soit 44 films sur 126) et sur les éléments que nous avons été à même de retrouver pour ce qui est du reste du corpus (résumés, photographies, articles de presse). Pour la commodité de lecture, nous ne ferons référence dans la suite du texte qu'à un petit nombre des films visionnés en indiquant le titre du film et la date de sa production. Nous les avons retenus parce que significatifs de la représentation managériale telle que nous avons pu l'apprécier au sein de ce corpus.

Dans la lignée de l'historien Pierre Sorlin (1977) et d'Alain Masson (1994) nous nous sommes attachée au film, à sa logique narrative. Nous avons cherché à comprendre son mode de fonctionnement prioritairement à l'étude du contexte de réalisation et de réception. En effet, nous avons considéré que les représentations sont à rechercher dans le film lui-même et non dans les éventuelles déclarations d'intention de ses auteurs quels qu'ils furent (scénaristes, réalisateurs, opérateurs, monteurs, producteurs, décorateurs, etc.).

\section{Une fonction minorée malgré le contexte favorable}

La modernité vient bousculer le lieu confiné du bureau salon du passé pour en faire un bureau spacieux, lumineux, situé dans les étages supérieurs de l'entreprise. On trouvera le goût pour ces vastes espaces dans des films réalisés entre la fin des années 1920 et le milieu des années 1930. Cet espace directorial s'accompagne parfois de grands bureaux collectifs vitrés où s'activent des dactylos alignées derrière des tables de travail et que traversent prestement des jeunes gens encravatés. On reconnaîtra là une scène d'Un fils d'Amérique - 1932, récit 
enthousiaste d'une «mécanisation » réussie dans l'industrie de la parfumerie. Le monde des bureaux n'est plus celui des rond-de-cuir, une nouvelle organisation du travail apparait et ce monde des employé(e)s s'étend aussi dans une industrie dont la représentation était jusque là davantage centrée sur les ateliers.

Autour du dirigeant, pour relayer la fonction administrative, on trouve, parfois, un ou une secrétaire, et des hommes en costume sombre. Cependant la définition d'une fonction administrative reste imprécise. S'agit-il d'un service administratif, « (...) sa constitution et ses attributions ne sont pas bien connues; ses opérations ne tombent pas sous les sens; on ne le voit ni bâtir, ni forger, ni vendre, ni acheter..., et cependant chacun sait que s'il ne fonctionne pas bien, l'entreprise périclite. » expliquait Fayol en 1900 (1999, p.128) alors qu'il tentait de convaincre la «sorte de franc-maçonnerie technique » (ibid.) en charge de la direction des entreprises.

L'image des cadres administratifs pour cette période tend à surenchérir le caractère malaisément définissable de leur rôle. La fonction est récente. A l'écran, elle apparaît dans les personnages d'hommes en costumes gris, présents dans l'environnement immédiat du directeur. Faute d'être bien définis, ils forment un groupe homogène que nous désignerons par le terme de cadre administratif dans la suite de ce texte. Les films en font un faible usage, ils forment comme un ballet autour du dirigeant quand ils sont visibles : jeunes gens passant et repassant dans le bureau du dirigeant, hommes en costume sombre dans les bureaux, ils ne sont pas nommés. Le pas vif, ils sentent la compétence des gens formés et assurés de leur place. Ils pourraient être employés aux écritures, adjoints ou bien même sous-directeurs ; la fiction s'autorise l'imprécision. Rien dans les récits ne permet au spectateur de mieux spécifier leur rôle. Un film de 1925, Amour et carburateur, dont l'action est située dans l'un des secteurs les plus dynamiques de l'économie française, la construction automobile (Verley, 1994, Fridenson, 1972), place ces personnages à l'arrière-plan d'une intrigue pourtant centrée 
sur l'entreprise. Nous, spectateur, ne saurons en quoi consiste leur rôle ; muets, ils restent de simples figurants filmés en plans moyens ou plans d'ensemble.

Une décennie plus tard, l'image se précise. Dans Si j'étais le patron en 1934, quand les ouvriers parlent des cadres administratifs, ils sont «les huiles, la direction, tout le bataclan» ${ }^{1}$, quand l'ingénieur désigne l'un d'entre eux, il dit «le sous-directeur », quand ils se rendent à la fête organisée par les ouvriers, ce sont des hommes en costume sombre, pochette blanche, qui restent entre eux évitant de «se commettre » avec les ouvriers. Ils constituent un groupe homogène, et l'intrigue ne permet guère de les distinguer les uns des autres. Nous ignorons tout de leur activité quotidienne. Pourtant, au début du récit, le dirigeant des Etablissements Leroy explique placidement à son Conseil d'administration que l'entreprise n'a ni bénéfice ni projet à leur présenter et propose une recapitalisation pour faire face à cette atonie. Le film ne montrant à aucun moment le rôle des cadres administratifs dans l'entreprise induit une déconnexion entre leur action et la marche de la firme. Cette conception restreinte du rôle du cadre est celle que retiennent nombre de fictions contemporaines : ces hommes peu accessibles, surtout affairés autour du dirigeant, sont aussi visibles dans A nous la liberté! 1931, Le Temps des cerises -1937, Adrien - 1943.

Tardivement leur rôle s'étoffe mais reste contraint à un registre limité : dans Au Bonheur des Dames en 1943, Octave Mouret, dirigeant fondateur, dispose d'un sous-directeur. Et, celui-ci a davantage un rôle d'alter ego que ses prédécesseurs. Dans l'entreprise commerciale qu'est le grand magasin, il est l'interlocuteur du directeur, son exécutant privilégié. Si Mouret peut être vu comme un dirigeant novateur, c'est qu'il se révèle à nous dans son dialogue avec son adjoint. Ce dernier a une vision terre à terre, n'anticipant pas les réactions de la clientèle, indifférent au sort des employés et soucieux avant tout de ce qu'il est «intéressé aux bénéfices ». Ainsi doté d'une personnalité opposée à celle de Mouret, il paraît cependant

\footnotetext{
${ }^{1}$ Citation extraite des dialogues du film. De même pour les suivantes.
} 
indispensable à la réflexion du dirigeant car investi du rôle de partenaire faire-valoir ; il devient réceptacle de la parole et de la pensée d'un autre mieux à même de concevoir.

Pour qui est à la recherche de l'image du cadre administratif, ce qui marque la période est surtout l'utilisation collective des hommes en costume gris. De l'ensemble des films visionnés, il nous reste l'image de la cour que ces hommes forment autour du dirigeant. Leur manière de faire masse autour du décideur renvoie à la dimension symbolique de leur fonction.

Rôle décoratif, fonction méconnue : voilà ce qui ressort des évocations proposées par un média de divertissement destiné à un large éventail de la population (Meusy, 2002). Pourtant, depuis les encouragements de Fayol, le discours sur la nécessité de la fonction administrative s'est nourri. Victor Cambon, Georges Lamirand, parmi d'autres, œuvrent à la diffusion du discours managérial auprès des ingénieurs. Le premier dans son enseignement à l'Ecole centrale des Arts et Manufactures qui fera l'objet d'une publication en 1920, le second par son ouvrage à la manière de Lyautey qui, en signant la préface à ce texte de 1932 destiné aux jeunes gens, marque lui-même la filiation entre la conception du rôle social de l'officier et celui de l'ingénieur. Tous deux, dans une logique didactique - pour les textes auxquels nous faisons référence -, développent un grand nombre d'exemples concrets. Lamirand met en scène les situations sur la base de saynètes et d'échanges dialogués qui nous rapprochent d'un cinéma devenu parlant depuis peu. Et si Cambon privilégie le modèle américain, on trouve chez Lamirand la référence à des collectifs nationaux œuvrant pour la diffusion des bonnes pratiques en matière d'organisation. Ainsi, sur un point de détail de son exposé, à propos du « respect des délais de livraisons », il note : « des organisations scientifiques du travail mènent depuis peu une énergique campagne de conférences et de tracts pour insister sur ce point. » (1932, p.48). Et parmi les commissions et groupements acteurs de l'institutionnalisation, citons pour mémoire le Comité National de l'Organisation Française 
(CNOF) et la Commission Générale de l'Organisation Scientifique du Travail (CGOST futur Cegos), organismes mis l'un et l'autre en place à partir de 1926.

Le courant est donc actif, audible dans diverses sphères de la société. Le cinéma, industrie de pointe, évolutive, n'y est pas hermétique. Le réalisateur Marcel L'Herbier lui-même dit combien le souffle de la rationalisation a su répondre à une attente dans sa pratique professionnelle et dans son rapport aux producteurs (Bertrand, 1937). Et c'est sans parler des industriels français qui, jusqu'à la fin des années 1920, tiennent une position dominante sur le secteur tels Léon Gaumont ou Charles Pathé (2006). Ces éléments laissent supposer que les films de fiction portant sur le monde du travail et l'entreprise sont assez précisément informés sur une réalité à laquelle ils entendent coller. Le corpus laisse ainsi entrevoir les préoccupations d'une époque ; la thématique de «la réduction des frais généraux », pour ne citer qu'un exemple, passe ainsi de film en film faisant écho aux types de réponses apportées par les entreprises à la crise des années 1930.

Dans ce contexte, la littérature de culture générale peut consacrer l'avènement d'une ère nouvelle : «L'ère des conquérants tend peu à peu à n'être plus que le passé. Nous arrivons à l'époque qu'on a pu appeler l'ère des techniciens de la direction, et ces techniciens sont aussi éloignés des ingénieurs et des capitalistes que des ouvriers. »(Palewski, 1928, p.248).

\section{Des légitimités en confrontation}

Dans ce contexte favorable, et pour comprendre la représentation restrictive des cadres administratifs, il est nécessaire de prendre du recul et de considérer dans son ensemble l'entreprise et son personnel.

Quelques mots de la direction générale avant de revenir à ce qui nous occupe vont permettre de comprendre ce qu'est l'entreprise racontée par la fiction. A travers les films, se laisse saisir une forme compartimentée assez similaire à la structure divisionnaire que des auteurs comme 
Alfred Chandler (1988) ou Henry Mintzberg (1989) ont rendue familière. La ligne hiérarchique très nette garde au dirigeant la prééminence. Des strates supplémentaires s'intercalent entre lui et la main-d'œuvre ouvrière: ce sont des services ou divisions regroupant une équipe d'ingénieurs, un ou des services techniques (dessinateurs industriels) ou administratifs (archives, comptabilité, relations avec le personnel). Ce sont aussi des niveaux hiérarchiques supplémentaires: surveillants, contrôleurs, chronométreurs, contremaîtres, chefs de bureau, chefs de rayon, directeurs adjoints. L'analyse des films met en relief la séparation entre propriété et direction de l'entreprise. Absente pour l'époque précédente des films que nous avons pu visionnés, cette séparation est ici très marquée. A l'exception de deux films, La Lutte pour la vie - 1914 et Amour et carburateur - 1925, la série laisse la place à un dirigeant dépendant de commanditaires ou d'un conseil d'administration.

Dans ce contexte d'évolution du capitalisme, la fiction offre une vision fort peu laudative des nouveaux spécialistes qu'évoquait plus haut Palewski. Le corpus témoigne de l'insistance portée sur les tensions qui traversent les firmes et en particulier sur la concurrence qui se joue entre les divers prétendants à la légitimité dans la firme. La fonction administrative semble envisagée sous la forme interrogative. Comment désigner celui qui est apte à tenir le rôle managérial est ce qui occupe les scénarios. Le cadre administratif n'est qu'un des postulants à la place laissée libre entre le détenteur de la firme et les ouvriers et employés. Notre corpus filmique révèle la grande diversité des acteurs qui se considèrent partie prenante du pouvoir de décision dans l'entreprise et les rapports complexes qu'ils entretiennent les uns avec les autres.

Au sein de l'entreprise même, la représentation distingue deux domaines : l'un relevant d'une compétence métier que nous pourrions dire technique, l'autre relevant de l'administration de l'entreprise. Il semble nécessaire de passer en revue les différentes compétences en 
commençant par la fonction administrative avant d'en examiner la légitimité. A ce domaine est rattaché un moindre nombre de personnages : autour du dirigeant, pour relayer la fonction administrative, on trouve un ou une secrétaire et le ballet des 'cadres administratifs'. Le secrétaire, proche du dirigeant, tout à la fois conseil, informateur, exécutant, tient la fonction d'éminence grise. L'interprétation par Antonin Artaud du secrétaire du banquier Saccard offre une image ambiguë de cette éminence grise. Par sa présence physique, il incarne un personnage aussi séduisant qu'insaisissable dans la Banque Universelle de L'Argent - 1928. Louis Jouvet interprètera un conseiller du prince au profil similaire dans Forfaiture - 1937.

Dans ce poste ou plutôt sous ce même intitulé, les femmes assurent un rôle d'exécutant dont on ne prise pas l'initiative. La secrétaire est assise derrière un bureau de petite taille, le secrétaire est mobile, allant du bureau du dirigeant à d'autres cénacles. Le corpus donne l'indice de la faible autonomie des femmes dans l'exercice d'une profession. Pour la firme Leroy dans Si j'étais le patron - 1934, Mireille Balin incarne la jolie secrétaire de Mr Leroy et semble une très sage « $M^{\text {elle }}$ Marcelle » derrière son petit bureau dans le hall adjacent le bureau de son patron. Quand son personnage interprète trop libéralement un ordre, il est immédiatement rappelé à l'ordre. Le commentaire d'un des cadres administratifs sera : «Mais c'est subversif. Si les subordonnés commencent à prendre des initiatives, alors où va-t-on ?» On voit ici que les porteurs de la fonction administrative autres que les cadres sont eux réduits aux types connus, voire aux stéréotypes. Notre corpus laisse paraître des fonctions simplifiées (les secrétaires) et des fonctions à l'occupation floue (les cadres administratifs) à côté de rôles plus nettement définis pour les postes à caractère technique. Ces figures porteuses d'une fonction technique sont plus nombreuses et plus élaborées : s'imposent en particulier les figures de contremaîtres, d'ingénieurs et d'inventeurs.

Le privilège accordé au domaine technique à travers ces trois personnages résonne dans la littérature managériale, que l'on se réfère au poids que lui ont conféré Henri Le Chatelier (in 
Vatin, 1990) ou Georges Lamirand. Le premier fait crédit à la capacité de Taylor d'intervenir pertinemment sur l'organisation du travail au vu de ses brevets, le second fait un constat identique : «L'ingénieur est d'abord un technicien. Il va sans dire qu'en progressant dans la hiérarchie l'importance de ses qualités techniques cédera le pas à celle de ses aptitudes administratives; mais son premier commandement étant un commandement d'atelier, c'est sur le terrain technique qu'il devra d'abord se faire juger. » (1932, p.34).

La légitimité technique ici ne relève pas du domaine de l'expertise au sens d'un savoir spécialisé, il s'agit bien davantage de l'exercice d'une capacité à conceptualiser de façon créative que le cinéma associe aux figures de l'inventeur et de l'homme de métier qu'il soit ouvrier ou ingénieur. Cette entrée technique dans la fonction managériale est susceptible d'être investie par des individus de toute origine sociale. Toujours dans notre corpus, c'est cette clef technique qui supporte l'essentiel de la dimension sociale du management. Quand Lamirand note: «Pour le capital, premier facteur de la production, l'usine apparaît donc bien comme une machine à fabriquer des dividendes. » (ibid., p.28), il propose à l'ingénieur d'investir le rôle de direction dans une dimension autre. Il attribue à cette dimension trois caractères : technique, administratif et social. Si le caractère technique est premier dans la carrière de l'ingénieur, Lamirand se réfère à Henri Fayol pour poser la prédominance de l'administratif dans la prise de direction. Dans ce cadre, le social est ce qui donnerait sens à l'action de l'ingénieur, le centrage sur les dividendes se traduisant pour l'ingénieur par le souci du prix de revient - préoccupation qu'il devra inscrire dans un regard humaniste sur l'usine. Dans ce texte d'invite faite au jeune ingénieur, est ainsi proposée une motivation en rupture avec la fabrique de dividendes.

Face au poids de ces hommes de métier, quelles sont les autres légitimités présentes à l'écran pouvant prétendre concilier le souci des hommes et celui des dividendes ? Le panorama contrasté offert par la fiction dévoile la coexistence de différents modes de fonctionnement et 
de différents ethos. A la vision saint-simonienne du Maître des forges - 1933 répond le questionnement de la valeur du travail - que soit abordée la vision du Front populaire sur la question (La vie est à nous - 1936) ou que soit fait témoignage de l'usure de la crédulité ouvrière (Le temps des cerises - 1937). La prodigalité comme mode de conduite de certains (Travail - 1919) côtoie la rigueur morale d'autres cadres ou dirigeants (La Lutte pour la vie 1914 ou Forfaiture - 1937). A l'interrogation : qui a son mot à dire dans l'entreprise? les films apportent une réponse plurielle. Les légitimités sont diverses. Pour le dire schématiquement, trois grandes formes de légitimité s'opposent et se contestent le droit d'intervenir sur le destin de l'entreprise : la compétence technique, la légitimité de l'organigramme et celle du capital. Et la légitimité par l'organigramme qui nous intéresse ici est comme constatée par la fiction pour être amplement moquée.

A l'instar des contremaîtres à l'autoritarisme partial, les directeurs et cadres administratifs qui ne disposent pas dans la fiction d'un bagage technique ne se voient dotés d'aucune compétence qui ait quelque épaisseur. Des diagrammes ornent les bureaux, des réunions se tiennent, mais pas plus la position dans la hiérarchie que le vernis administratif ne convainquent. Les porteurs d'une compétence administrative peinent à exister dans la fiction, l'action des hommes de bureau reste transparente, impossible à valoriser et donc trop fragile pour supporter une quelconque légitimité. Et le capital ne semble pas plus porteur d'une légitimité managériale. Les films ne jouent pas tant le capitalisme financier contre le capitalisme patrimonial dans la question managériale. Entendons-nous, ces deux formes de capitalistes sont distinctes dans le corpus, mais se rejoignent en ce qu'elles ne sont pas porteuses d'une légitimité à diriger l'entreprise. Le projet dévolu par les récits à l'entreprise dépasse très largement le champ de la motivation de l'actionnaire spéculateur à courtes vues, du patriarche appartenant à un ordre ancien dont les règles n'ont plus cours ou de l'héritier prodigue. Par ailleurs, l'exogamie des héritières, tellement affichée par le cinéma, nous dit, en 
creux, quelque chose de l'insuffisance prêtée à la lignée. Les filles d'industriel n'épousent pas plus un héritier qu'un cadre administratif dans la fiction, elles convolent avec un ouvrier, un contremaître, un inventeur, un ingénieur. Le capitalisme patrimonial trouve une redynamisation dans l'exogamie, les films ne vont pas au-delà de la scène nuptiale et ne disent pas si cet apport assure la pérennité des entreprises familiales.

La fiction pare l'un et l'autre capitalisme de caractéristiques ambivalentes, assez souvent défavorables, et donne le privilège à une compétence présentée comme nouvelle : il s'agit de la compétence technique que nous avons décrite plus haut et qu'il faut entendre au sens de métier plus qu'au sens d'expertise. Un bon manager ne peut pas être qu'un homme de bureau, il n'est pas non plus cet homme de l'entre-deux qu'est parfois le contremaître à l'écran, il est bien plutôt un individu mobile, capable de passer de l'atelier au bureau, apte à prendre en main des situations diverses : la confrontation avec les grévistes, l'accident, le conseil d'administration houleux, les pressions politiques, le salon de réception... Les hommes de la gestion valorisés dans ce corpus de films se définissent par leur capacité à la mobilité. Le parcours de la société peut être entendu ici comme le fait d'échapper à la pesanteur d'un ordre social. Mais le refus du déterminisme n'épuise pas ce parcours, les films montrent moins l'ascension d'un individu que la reconnaissance de sa valeur et, chez lui, une forme de fidélité à son origine sociale ou tout au moins la reconnaissance des ouvriers ou employés comme des interlocuteurs possibles. Par ce dialogue autorisé, encouragé, et par le franchissement des barrières sociales, il se démarque profondément de ses compétiteurs. Qui est-il ? L'ouvrier inventeur, le technicien entreprenant, l'autodidacte réalisateur, en un mot le dirigeant légitime. C'est un concepteur immergé dans le collectif de production, il apparaît nettement comme une figure morale dont la motivation justifie l'élection. Et il est l'homme de la mobilité. Cette qualité garante d'efficacité est déniée aux cadres administratifs. 


\section{Le costume empesé du cadre}

Les cadres, les adjoints et les sous-directeurs de cinéma revêtent une qualité qui les sort du jeu ; ils endossent leur fonction comme un état. Comment cet immobilisme apparaît-il à l'écran ? Il est visible dans les caractéristiques associées aux cadres mais aussi à travers les récits qui en usent comme de silhouettes fortement typées. Dans les mondes professionnels que les fictions créent sous nos yeux, les caractères physiques des cadres se font l'écho de leurs compétences.

Les personnages assimilés aux cadres n'occupent pas le devant de la scène, ils sont représentés en tant que seconds rôles. Il s'agit le plus souvent d'un collectif qui sert de fairevaloir au dirigeant et d'où n'émerge pas d'individualité qui retiendrait l'intérêt du spectateur (Amour et carburateur - 1925, A nous la liberté - 1931). Engoncés dans leurs costumes, ils semblent peu capables d'agir dès lors que l'on prête attention aux récits : L'Aventurier - 1934 montre un responsable administratif incapable de contrer et l'héritier dispendieux et les ouvriers grévistes. Seule une intervention extérieure permettra à la firme de surmonter la crise. La même année, Si j'étais le patron moque des cadres plus soucieux de leurs prérogatives que du bilan exécrable de leur firme. Cette disjonction entre les cadres administratifs et l'organisation est loin de faire exception. La présence des cadres est fréquemment déconnectée de la bonne marche d'une entreprise dont ils ignorent un grand nombre d'aspects ; l'innovation technologique (A nous la liberté - 1931) ou commerciale (Au Bonheur des Dames - 1943) leur échappe. Ils veulent ignorer les contraintes d'un projet d'envergure (Forfaiture - 1937) ou préfèrent ne rien savoir des exécutants (Le Temps des cerises - 1937) ; ils suivent le mouvement dominant sans tenter une opinion divergente (L'Argent - 1928, L'Embuscade - 1939). Visibles dans l'entreprise, ils n'ont pourtant pas de lieux à eux : le dirigeant dispose d'un vaste bureau, l'ingénieur est près de l'atelier. Dans plusieurs fictions, les cadres administratifs sont montrés formant une cour autour du dirigeant. 
L'incapacité à l'action des cadres administratifs est ainsi perçue par le spectateur de la fiction comme le corollaire de leur place décorative. Ces personnages paraissent statufiés à l'écran. Statiques, ils ne sont pas à même d'intervenir dans l'entreprise par une démarche novatrice. Le risque de l'erreur ou de l'échec paraît alors la justification de leur inaction. L'expérimentation est exclue quand le droit à l'erreur ne fait pas partie de la panoplie et quand la séparation entre usine et bureaux est étanche. Ce qui serait en jeu dans l'engagement serait leur statut distinctif qui repose sur une position figée. L'échec possible, anticipé, devient un trop grand risque pour leur permettre de s'engager dans l'action.

Cette représentation contraste fortement avec les textes des théoriciens, français ou nord américains, qui lui sont contemporains. En dépit de toutes leurs divergences, ces penseurs de la fonction administrative ont pour point commun la revendication d'une nécessaire expérimentation dans l'organisation. La souplesse d'intervention que cela suppose trouve une définition changeante selon les doctrines. Ainsi, Henri Fayol n'a de cesse de souligner que sa doctrine administrative n'est pas un dogme, mais repose sur le sens de la mesure. Ce n'est pas trahir sa pensée que d'entendre cette caractéristique comme une forme d'adaptabilité constitutive des modes de fonctionnement de l'entreprise. Il dessine ainsi une entreprise aux contours fluctuants en fonction de l'intérêt de l'organisation, de ses spécificités et des circonstances. La souplesse d'intervention se joue pour Fayol dans l'exercice même de la fonction managériale : «Il n'y a rien de rigide ni d'absolu en matière administrative ; tout y est question de mesure. » (Fayol, 1999, p.23)

Si l'on trouve chez Taylor la même volonté d'application universelle de la doctrine que chez Fayol (dans l'usine, dans la sphère publique et dans la famille), il se distingue de ce dernier par son terrain d'observation. Fayol, ingénieur de l'Ecole des Mines de St-Etienne, a exercé des fonctions de direction dans la société minière Commentry-Fourchambault, quand Taylor (1993) a conçu sa doctrine sur une connaissance au plus proche du monde de l'atelier et par 
l'expérimentation sur le travail des manœuvres. Les différences ne s'arrêtent pas au lieu de l'observation et concernent les prescriptions : Taylor propose une norme scientifique dont on ne saurait dévier sous peine d'échec, son compétiteur français propose une culture administrative qui est une culture du management adaptative du fait de la soumission à l'intérêt général et de la place donnée à l'initiative individuelle. Malgré leurs divergences que Fayol n'a pas manqué de souligner, reste chez Taylor l'idée d'une expérimentation nécessaire à la conception et à la mise en œuvre progressive de l'organisation enfin rationnelle. Pour Taylor, l'expérimentation appartient à l'expert en management et s'exprime dans la dimension temporelle de la démarche: formation progressive des manœuvres un par un, essais permettant de parvenir à définir une norme standard...

Mais, à l'instar de Fayol, s'il est un auteur américain qui se fit l'avocat infatigable du management comme discipline à même d'être enseignée et comme ensemble de compétences à expérimenter, c'est bien Mary Parker Follett. Dans la seconde moitié des années 1920, elle multiplia conférences et publications aux Etats-Unis et en Grande-Bretagne à destination des dirigeants d'entreprises. Si ses textes ont été traduits tardivement en français, il nous semble cependant pertinent de nous tourner vers sa pensée afin d'apprécier un point de vue contemporain sur ce que devait être la fonction administrative : "Managerial skill cannot be painted on the outside of executives; it has to go deeper than that. Like manual workers, managerial workers have to acquire certain habits and attitudes. And, just as in the case of manual workers, for the acquisition of these habits and attitudes three conditions must be given: (1) detailed information in regard to a new method; (2) the stimulus to adopt this method; and (3) the opportunity to practice it so that it may become a habit.» (in Owen, 1993, p.130). Le vernis auquel Follett fait allusion dans cette citation semble le terme approprié pour décrire les cadres administratifs de la fiction contemporaine. Follett, en soulignant la nécessité de l'expérimentation organisationnelle, exprime une vue intéressante qui va au-delà 
de la nécessité de la formation au management demandé par Fayol : le management s'apprend dans une démarche autorisant essais autant qu'erreurs dans la mesure où le cadre est aussi capable de tirer un enseignement constructif de ses éventuels échecs.

Les textes auxquels nous nous sommes référée s'étalent sur un quart de siècle et émanent de deux cultures différentes, cependant la demande d'un management éclairé infuse cet ensemble. Tous dessinent l'image de ce qu'ils considèrent être un management avisé et qui devrait se généraliser sous peu. La persistance de la promesse laisse imaginer un décalage entre la réalité décrite/voulue par les théoriciens et celle vécue par les praticiens. Elle contraste, par ailleurs, avec l'imagier du cinéma qui fait des cadres administratifs des répliques amoindries du dirigeant. La question face aux personnages de cadres administratifs de cinéma, ces hommes en gris indistincts, est : s'agit-il bien de management ? La réponse est affirmative par un effet de tautologie, ces cadres administratifs assurent une fonction managériale dans la mesure où ils sont de façon assertive et ostentatoire en situation de signifier leur statut. La réponse est négative si l'on en juge par la remise en cause de leur absence d'action dans la fiction. Ils sont contestés par les autres personnages du récit et par la narration elle-même qui réduit leur rôle à de la représentation en les cantonnant à l'arrièreplan.

Nous voudrions proposer une synthèse en nous attachant aux deux positions les plus typées de l'exercice de la fonction managériale. Ce choix de mettre face à face le cadre administratif ou 'sous-directeur' et l'ingénieur correspond à la représentation bipolaire du management que privilégie le cinéma français. S'il est bien une constante dans cette part du récit national, c'est la tension entre deux manières d'habiter le rôle de manager - tension sensible dans la totalité du corpus qui nous occupe ici. La figure 1, ci-après, rend compte de cette figuration tirée vers une logique de confrontation duelle. 
Figure $n^{\circ} 1$ - Les attributs managériaux

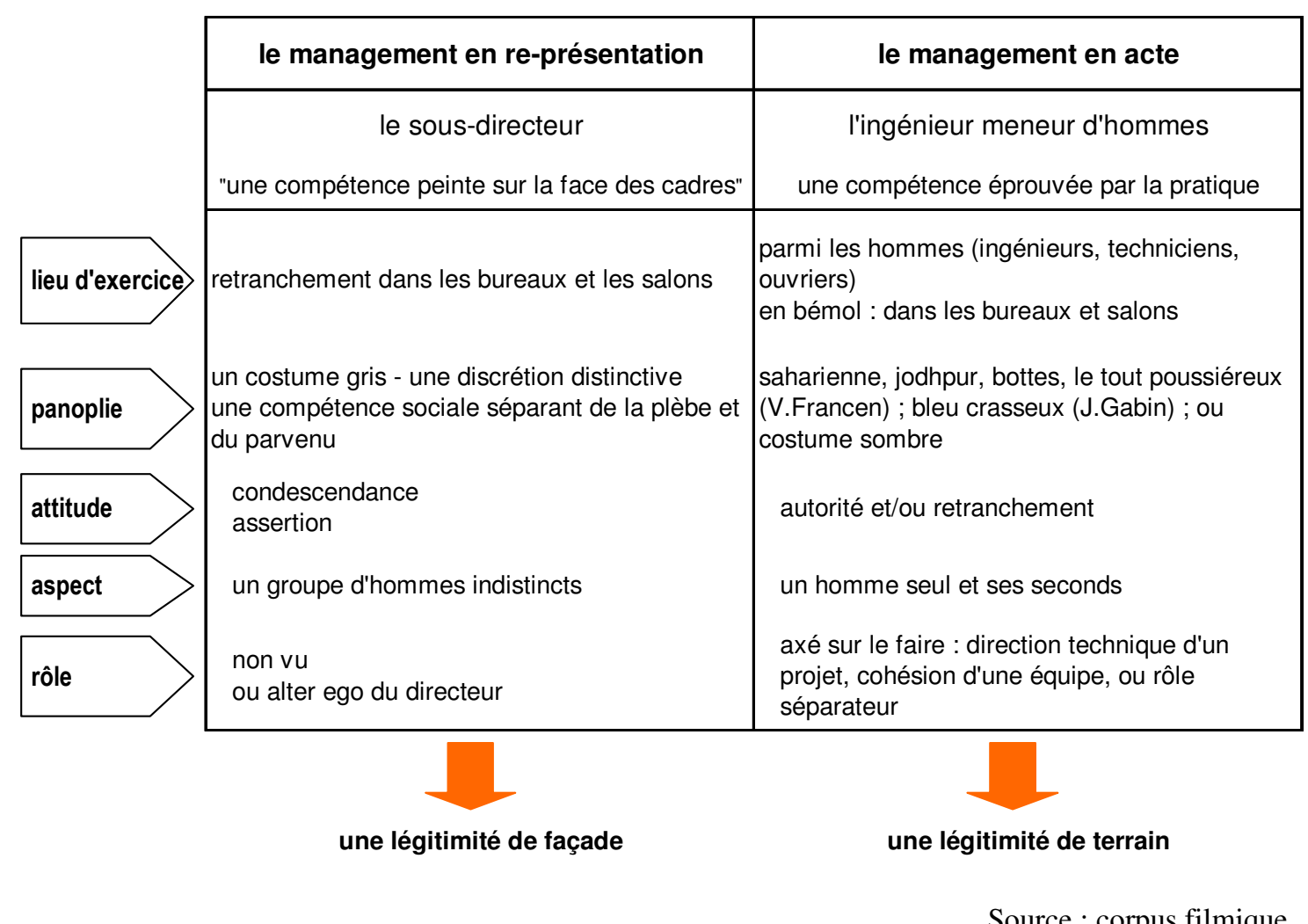

Les fictions, en favorisant les personnages à compétence technicienne pour représenter les hommes du management, donnent plus de poids à certains professionnels : individualisés, ce sont les inventeurs, les ingénieurs souvent autodidactes mais aussi sortis des écoles, voire les contremaîtres. Qu'ils soient héros du récit, faire-valoir, simples comparses, qu'ils tiennent le rôle du vilain, leurs personnages sont nourris, fouillés. Nous les voyons à l'œuvre quand les hommes en costume gris restent un ensemble indistinct. Tout réduit la compétence de ces derniers à un savoir être d'ordre social témoignant d'une parfaite maîtrise de la bienséance : bien se vêtir, savoir se tenir dans le monde, donner des ordres aux domestiques.

La légitimité dans la fiction repose sur la capacité à cumuler des capacités techniques et sociales spécifiques. L’idéaltype managérial émergent repose sur un individu qui se positionne dans le mouvement, se construit par le dialogue et conçoit des agencements nouveaux qu'ils soient techniques ou sociaux. Toutes choses que le cadre administratif ne peut se permettre. 


\section{Conclusion : Quand nommer et représenter, c'est penser}

La possibilité de se former à la fonction administrative professée par Follett comme par Fayol bute contre la représentation d'une activité professionnelle pensée comme un état que l'on endosse une fois pour toute. Dans ses écrits et conférences des années 1920, Follett professe l'expérimentation comme méthode de base pour le management. Elle souligne inlassablement la nécessité de l'engagement auprès des responsables d'entreprise : «Nous ne pouvons pas regarder une expérience sans en faire partie. La vie n'est pas un film pour nous; nous ne pouvons jamais être spectateurs de la vie, parce que nous sommes toujours dans la vie.» (2002, p.43). Dans la perspective de Fayol ou de Follett, la peur de l'échec est un plus grand échec que les erreurs inhérentes à une approche expérimentale de l'administration des entreprises. Et pourtant, l'image marquante véhiculée par un média de masse dans la société française contemporaine est celle d'une profession déjà rigidifiée. Le cadre, cantonné à un rôle de représentation, voit toute capacité à agir lui échapper. La fonction du cadre semble alors en concordance avec son nom même, le 'cadre' accroché une fois pour toute à la vue de tous.

La représentation filmique n'est ni la réalité de l'entreprise, ni son reflet. Nous avons choisi de nous y intéresser car nous considérons que les représentations donnent à voir la manière dont une époque se pense. Et dans le temps que nous avons considéré, il apparait que la dimension de conceptualisation et d'expérimentation associée au manager idéal est déniée au cadre administratif. Le manager idéal est non seulement celui qui est efficace (l'efficacité étant définie en termes financiers et/ou en termes de production : Travail - 1919, L'Aventurier - 1924 et 1934, Si j'étais le patron - 1934...), il est aussi celui qui concilie l'exigence comptable au souci des hommes. Cette représentation privilégiée apparaît comme une manière de s'approprier comme un souffle revigorant la culture de la méritocratie. «En 1911, 75\% d'ingénieurs, en 1937, 82\%, la plupart sortis de Polytechnique ou de Centrale, 
gouvernent au nom des actionnaires. Cette 'méritocratie' entraîne une légère ouverture des classes dominantes. Vers 1930, environ $12 \%$ du groupe des dirigeants peuvent revendiquer une origine populaire. » (Woronoff, 2003, p.20).

Ces chiffres montrent la survalorisation du mouvement méritocratique dans le récit cinématographique et laissent apprécier par contraste la nécessité dont la fiction pare cette ouverture quand le statut du cadre le maintient à distance d'une vie à laquelle il est étranger, car il reste invisible dans l'atelier, parmi les pools de dactylos et les employés, parmi les clients dans les grands magasins ou sur les boulevards. On voit ainsi se nourrir le soupçon à l'encontre d'une profession récente et encore jugée superflue. Le statut offert par le titre de cadre ne servirait que les intérêts de l'individu qui en est porteur. Et cela apparaît dans le cinéma de la période 1914-1947 comme une proposition inadmissible quand les théoriciens de l'organisation ainsi que les films de fiction font de l'entreprise un projet qui dépasse les hommes, et ce, bien que la filmographie de notre période ne soit pas un ensemble homogène et statique. L'absence de crédit accordé au cadre administratif dans la fiction de ces années réduit le rôle au port de costume et la représentation symbolique n'est pas une des fonctions du cadre mais son seul mode d'existence dans l'entreprise. A l'écran, la prise de fonction du cadre est montrée comme une finalité et non comme une entrée en matière pour agir sur l'entreprise. L'attachement à un statut prévaut à l'engagement dans l'action.

Le décalage entre les images filmiques et le discours théoricien sur le management qui leur est contemporain semble faire état d'une concurrence à penser les rapports de travail. Dans la littérature de gestion, associer au cadre une fonction symbolique de représentation apparaît tardivement ; la revendication se joue au-delà de la période qui nous intéresse, et, l'ouvrage de Mintzberg (Le manager au quotidien, 2005) originellement publié en 1973, en serait une des premières expressions. La fiction paraît ainsi un champ d'investigation fécond pour la pensée gestionnaire : le récit au cinéma saisit des éléments qui échappent au théoricien. Ces 
éléments sont les modes de penser le contemporain propre à une société donnée, il s'agit parfois d'évidences que seul un candide est susceptible d'interroger, mais que les films véhiculent et répètent au fil de leurs diffusions et rediffusions. Par l'étude des représentations dont les films sont porteurs, nous pouvons trouver, en tant que gestionnaire, une mise en perspective permettant de questionner les résistances à l'exercice de la fonction administrative hier et aujourd'hui. Si le vocabulaire évolue, si l'on est passé du cadre administratif au manager, la fonction continue à faire question. 


\section{Bibliographie}

J. Bertrand, "Nos grands metteurs en scène", L’Epoque, 30 août 1937.

M. Bloch, Apologie pour l'histoire ou Métier d'historien, Armand Colin, Paris, 1967.

L. Boltanski, Les cadres. La formation d'un groupe social, Les Editions de Minuit, Paris, 1982.

V. Cambon, L'industrie organisée d'après les méthodes américaines, Payot, Paris, 1920.

A.D. Chandler, La main visible des managers, Economica, Paris, 1988.

H. Fayol, Administration industrielle et générale, Dunod, Paris, 1999.

M.P. Follett, Diriger au-delà du conflit: six conférences, Village Mondial, Paris, 2002.

P. Fridenson, Histoire des usines Renault. 1. Naissance de la grande entreprise : 1898 - 1939, Seuil, Paris, 1972.

G. Lamirand, Le rôle social de l'ingénieur, Ed. de la Revue des Jeunes, Paris, 1932.

A. Masson, Le récit au cinéma, Editions de l'Etoile / Cahiers du cinéma, Paris, 1994.

J.-J. Meusy, Paris-Palaces ou le temps des cinémas (1894-1918), CNRS Editions, Paris, 2002

H. Mintzberg, Structure et dynamique des organisations, Ed. d'Organisation, Paris, 1989.

H. Mintzberg, Le manager au quotidien. Les dix rôles du cadre, Ed. d'Organisation, Paris, 2005.

R. Owen, H. Metcalfe, J.S. Lewis, R. Robb, H.R. Towne, H.L. Gantt, M.P. Follett et H. Fayol, Classics of modern management theory, Routledge/Thoemmes Press, Londres, 1993.

J.-P. Palewski, Histoire des chefs d'entreprise, Librairie Gallimard NRF, Paris, 1928.

C. Pathé, Ecrits autobiographiques, L'Harmattan, Paris, 2006.

P. Sorlin, Sociologie du cinéma. Ouverture pour l'histoire de demain, Aubier Montaigne, Paris, 1977.

F. W. Taylor, The Principles of Scientific Management and Shop Management, Routledge/Thoemmes Press, Londres, 1993.

F. Vatin (dir.), Organisation du travail et économie des entreprises. 'Direction des ateliers' et le débat sur le taylorisme en France entre 1910 et 1920, Ed. d'Organisation, Paris, 1990.

P. Verley, Entreprises et entrepreneurs du XVIIIe siècle au début du XXe siècle, Hachette, Paris, 1994.

D. Woronoff, La France industrielle. Gens des ateliers et des usines, 1890-1950, Ed. du Chêne, Paris, 2003.

\section{Sources}

Catalogues généraux :

M. Bessy, R. Chirat, Histoire du cinéma français, Encyclopédie des films 1940-1950, Paris, Pygmalion, 1986.

R. Chirat, Catalogue des films français de long métrage, films sonores de fiction 1929-1939, La Cinémathèque Royale, Bruxelles, 1981.

R. Chirat et R. Icart, Catalogue des films français de long métrage, films de fiction 1919-1929, La Cinémathèque de Toulouse, Toulouse, 1984.

R. Chirat et E. Le Roy, Catalogue des films français de fiction 1908 à 1918, La Cinémathèque française, Musée du cinéma, Paris, 1995.

Catalogues par producteur:

Catalogue Gaumont. Index des sujets et des personnages (1906-1926), Bibliothèque Nationale, Département des Arts du spectacle, Paris, 1994.

H. Bousquet, De Pathé Frères à Pathé Cinéma, Edition Henri Bousquet, Paris, 3 volumes, 1999, 2001 et 2004.

P. d'Hugues et D. Muller (dir.), Gaumont, 90 ans de cinéma, Ramsay, La Cinémathèque Française, Paris, 1986.

E. Le Roy et L. Billia (dir), Eclair. Un siècle de cinéma à Epinay-sur-Seine, Calmann-Lévy, Paris, 1995.

B. Salomon, La société française des films et cinématographes Eclair de 1907 à 1918, Université Paris IV, Paris, mémoire de maîtrise, 1987. 
Autre :

S. Sakhno, Dictionnaire Russe Français d'étymologie comparée, L’Harmattan, Paris, 2001.

Origine des films visionnés (en dehors du circuit commercial des salles et éditeurs dvd ou vhs) : Archives du Film - CNC, Archives Gaumont-Pathé, Bifi, Bnf, Cinémathèque Française, Cinémathèque Ledoux, Forum des Images.

\section{Filmographie sélective}

(liste chronologique des films visionnés [Année de production - Titre du film - Réalisateur])

1914 - La Lutte pour la vie / La Vertu récompensée - F. Zecca \& R. Leprince

1914 - Rigadin mauvais ouvrier - G. Monca

1918 - Rigadin aimé de sa dactylo - G. Monca

1919 - Travail - H. Pouctal

1921 - Le Porion - G. Champavert

1921 - L'Empereur des pauvres - R. Leprince

1924 - L'Aventurier - M. Mariaud \& L. Osmont

1925 - Amour et carburateur - P. Colombier

1926 - Antoinette Sabrier - G. Dulac

1927 - Le Bateau de verre - C. J. David

1928 - L'Argent - M. L'Herbier

1931 - A nous la liberté - R. Clair

1932 - Théodore et Cie - P. Colombier

1932 - Un fils d'Amérique - C. Gallone

1933 - Ces messieurs de la Santé - P. Colombier

1933 - Le Maître de forges - F. Rivers \& A. Gance

1933 - Le Tunnel - K. Bernhardt

1934 - La Banque Némo - M. Viel

1934 - L'Aventurier - M. L'Herbier

1934 - Si j'étais le patron - R. Pottier

1934 - Toni - J. Renoir

1935 - Le crime de Monsieur Lange - J. Renoir

1936 - La Vie est à nous - J. Renoir, J.-P. Le Chanois, J. Becker, A. Zwobada

1936 - L'Argent - P. Billon

1936 - Les Hommes nouveaux - M. L'Herbier

1937 - Forfaiture - M. L'Herbier

1937 - Hercule - A. Esway \& C. Rim

1937 - Le Schpountz - M. Pagnol

1937 - Le Temps des cerises - J.-P. Le Chanois

1937 - Monsieur Bégonia - A. Hugon

1938 - Carrefour - K. Bernhardt

1939 - L'Embuscade - F. Rivers

1941 - Fromont jeune et Risler aîné - L. Mathot

1942 - Les affaires sont les affaires - J. Dréville

1943 - Adrien - Fernandel

1943 - Au Bonheur des Dames - A. Cayatte

1943 - Coup de tête - R. Le Hénaff

1943 - Le Val d'enfer - M. Tourneur

1945 - Etoile sans lumière - M. Blistène

1946 - L'arche de Noé - H. Jacques

1946 - Le silence est d'or - R. Clair

1946 - Martin Roumagnac - G. Lacombe

1946 - Miroir - R. Lamy

1946 - Voyage-surprise - P. Prévert 